\title{
Mechanism of ECM-induced dormancy and chemoresistance in A549 human lung carcinoma cells
}

\author{
SIRIPORN KEERATICHAMROEN ${ }^{1}$, KRIENGSAK LIRDPRAPAMONGKOL $^{1}$ and JISNUSON SVASTI $^{1,2}$ \\ ${ }^{1}$ Laboratory of Biochemistry, Chulabhorn Research Institute; ${ }^{2}$ Applied Biological Sciences Program, \\ Chulabhorn Graduate Institute, Bangkok 10210, Thailand
}

Received July 17, 2017; Accepted January 23, 2018

DOI: $10.3892 /$ or.2018.6258

\begin{abstract}
It is now widely accepted that the tumor microenvironment influences the fate of cancer cells and plays crucial roles in regulating tumor dormancy and chemoresistance. The standard cell culture system on plastic surfaces does not account for cell interactions with the extracellular matrix (ECM), and is thus a less reliable approach to analyze cellular activity ex vivo. In the present study, A549 lung cancer cells were cultured in a semi-solid growth substrate (Matrigel) to mimic the tumor microenvironment and to investigate the role played by ECM proteins, as well as to evaluate the mechanism of cell-ECM communication. A549 cells embedded in semisolid Matrigel exhibited dormant cell characteristics, including decreased cell proliferation, migration and invasion rates, compared with the corresponding cells cultured on plastic plates. Exposure of A549 cells to Matrigel leads to resistance against conventional chemotherapeutic drugs (etoposide, paclitaxel, vinblastine, doxorubicin and 2-deoxy-D-glucose). Cell cycle distribution analysis indicated that a larger percentage of the cells embedded within semi-solid Matrigel was arrested in the G0/G1 phase. RT-qPCR analysis revealed that A549 cells cultured in semi-solid Matrigel exhibited a marked decrease in the expression levels of genes that are related to tumor progression and invasion (UPA, uPAR, MMP2, MMP7, MMP9 and CXCR4). The effects of altering various signaling pathways, such as p-ERK, p-Akt and p-STAT3, were evaluated, in order to assess whether these pathways could account for the observed responses of the cells. The inhibition of ERK1/2 and Akt activation using specific inhibitors induced G0/G1 arrest and drug resistance. These results demonstrated that Matrigel drove A549 cells into a drug-resistant dormancy state, most likely through inhibition of the ERK1/2 and PI3K/Akt pathways. Cell culture within semi-solid Matrigel offered a simple
\end{abstract}

Correspondence to: Professor Jisnuson Svasti, Laboratory of Biochemistry, Chulabhorn Research Institute, 54 Kamphaeng Phet 6 Road, Talat Bang Khen, Laksi, Bangkok 10210, Thailand

E-mail: jisnuson@cri.or.th

Key words: metastasis, disseminated tumor cells, dormancy, chemoresistance, Matrigel, lung cancer in vitro model for studying the mechanisms responsible for tumor dormancy and drug resistance. These studies may lead to therapeutic approaches that can eliminate dormant tumor cells and prevent disease recurrence.

\section{Introduction}

An increasing body of evidence suggests that the tumor microenvironment has a profound impact on cancer progression and therapeutic responses (1-4). Extracellular matrix (ECM), a major component of the microenvironment, is composed of a combination of macromolecules (e.g. collagens, laminin, elastin, fibronectin, proteoglycans and polysaccharides). Cellular attachment to ECM components triggers intracellular signaling pathways that regulate numerous cellular functions, including cell proliferation, differentiation, cell shape, survival, migration and invasion (1,5). Cell-ECM interactions can influence patterns of tissue-specific gene expression, leading to different biological phenotypes. For instance, exposure of mammary epithelial cells to a laminin-rich basement membrane has been revealed to induce milk protein (beta-casein) expression (6,7). Factors in the tissue microenvironment have been suggested to influence the expression of ECM-degrading enzymes, thus modifying the ability of human colon carcinoma cells to metastasize at two different implantation sites (8). A previous study demonstrated the role of the microenvironment in the expression of urokinase-type plasminogen activator (uPA), and thus in the metastatic potential of KG-2 human renal carcinoma cells (9).

Metastasis, the spread of cancer to distant locations in the body, is a primary cause of cancer-related deaths. It has previously been reported that disseminated tumor cells can lie dormant in secondary organs for prolonged periods of time $(10,11)$. These quiescent dormant cells remain undetectable to screening methodologies and evade conventional therapies that target actively proliferating cells (12). Recent studies have demonstrated that the dormant cells can switch to proliferative metastatic growth when conditions are favorable, and thus may be responsible for disease recurrence (13). Tumor dormancy, chemotherapy resistance and cancer recurrence are major problems associated with cancer treatment. Components of the microenvironment have been reported to play a central role in regulating tumor dormancy and chemoresistance $(1,14,15)$. Elucidating the mechanisms underlying 
ECM-mediated tumor dormancy and the switch to metastatic growth should provide useful targets for the development of therapeutic approaches to eliminate these inactive tumor cells. This would reduce the risk of dormant cells becoming proliferate after long periods.

The microenvironment of cells cultured in vitro on traditional plastic substrata differs considerably from that of cells in vivo. Cells require not only nutrients and growth factors, but also appropriate interaction with ECM components in order to trigger intracellular signal transduction. Critical signals are lost when cells are cultured on plastic surfaces. Recent experiments revealed that most cell lines can readily proliferate as a monolayer on plastic plates, but do not develop tumors in vivo (11). Moreover, standard cell culture on plastic surfaces fails to predict realistic responses to drug candidates. However, most research has been performed with cancer cells grown on plastic substrata. Thus, the development of a cell culture system that recreates an appropriate environment for cells should provide a better model for studies of cellular activity.

Matrigel is a biological ECM extracted from mouse Englebreth-Holm-Swarm (EHS) sarcoma, a tumor that is rich in basement membrane and ECM proteins. Matrigel is composed of laminin, collagen IV, entactin, heparan sulfate proteoglycans, nidogens and a small quantity of protease, as well as growth factors (16). Matrigel polymerizes at room temperature to form a matrix material resembling the basement membrane found in many tissues. It is commonly used as a substrate for cell culture (17). In the present study, human non-small cell lung cancer (NSCLC) A549 cells were embedded in a semi-solid Matrigel matrix to enable close interaction with ECM proteins, a method that better imitates the in vivo microenvironment compared with standard cell culture on plastic plates. Cancer cell behaviors, including cell migration, invasion, chemosensitivity and proliferation, were assessed. In addition, the signaling pathways mediating the effect of Matrigel were studied to assess the molecular mechanisms involved in tumor dormancy and drug resistance.

\section{Materials and methods}

Cell culture. Human A549 NSCLC cells were purchased from the American Type Culture Collection (Rockville, MD, USA). Cells were maintained in RPMI-1640 supplemented with $10 \%$ fetal bovine serum and $1 \%$ antibiotic-antimycotic solution (Gibco; Thermo Fisher Scientific, Inc., Grand Island, NY, USA). For semi-solid Matrigel-embedded cell culture, a cell suspension ( $3 \times 10^{5}$ cells in $350 \mu \mathrm{l}$ of complete medium) was gently mixed with $150 \mu \mathrm{l}$ of Matrigel (containing growth factors) (Corning Life Sciences, Bedford, MA, USA), with a final concentration of $30 \% \mathrm{v} / \mathrm{v}$ Matrigel. This was plated into a 24-well plate and incubated at $37^{\circ} \mathrm{C}$ for $30 \mathrm{~min}$. Then, $500 \mu \mathrm{l}$ of complete media was gently added to the well and the culture was maintained for 3 days. This diluted Matrigel polymerizes at $37^{\circ} \mathrm{C}$ to form a semi-solid gel that is soft enough to allow cells to spread as a monolayer beneath the gel. Cells were harvested from the semi-solid culture environment, for further analysis of cell behavior, by tapping the plate, aspirating the collapsed gel and washing with PBS. This was followed by conventional trypsinization. Cell morphology was examined by inverted phase-contrast microscopy. In all experiments, the cells were maintained at $37^{\circ} \mathrm{C}$ in a humidified atmosphere containing $5 \% \mathrm{CO}_{2}$.

Cell proliferation assay. Cell proliferation was determined using the Quant-iT ${ }^{\mathrm{TM}}$ PicoGreen ${ }^{\circledR}$ dsDNA Assay kit (Invitrogen; Thermo Fisher Scientific, Inc., San Diego, CA, USA) to quantify DNA, according to the manufacturer's protocol. After harvesting cells from standard cell culture or semi-solid Matrigel-embedded cell culture, the cells were seeded into 96-well plates at a density of $1 \times 10^{4}$ cells/well in $100 \mu \mathrm{l}$ of culture medium. Cell proliferation was assessed on days 0, 2, 4, 7 and 9 after plating. Fluorescencebased dsDNA measurements were obtained using a SpectraMax fluorescence microplate reader (Molecular Devices, LLC, Sunnyvale, CA, USA), with an excitation wavelength of $485 \mathrm{~nm}$ and an emission wavelength of $520 \mathrm{~nm}$.

Cell migration (wound-healing) assay. Cell migration was assessed using a wound-healing assay. Wounds were generated by scratching confluent cultures of cells on plastic plates, or cells in semi-solid Matrigel (after removing the gel and washing with PBS), with a sterile $20-\mu 1$ pipette tip. These wounds were then washed twice with PBS to remove cell debris, covered with complete medium or $30 \% \mathrm{v} / \mathrm{v}$ Matrigel, and incubated for a further $24 \mathrm{~h}$. Wounds were marked and digitally photographed in the same field at 0 and $24 \mathrm{~h}$ after scratching. The wound area was assessed with ImageJ software, and the extent of healing was calculated as follows:

$\%$ Wound closure $=($ wound area at $0 \mathrm{~h}-$ wound area at $24 \mathrm{~h}) /$ wound area at $0 \mathrm{~h} \times 100 \%$

Invasion assay. The invasiveness of cancer cells was evaluated using Matrigel-coated Transwell chambers ( $8 \mu \mathrm{m}$ pore size). The upper chamber was seeded with $1 \times 10^{5}$ cells in a $200-\mu 1$ suspension, while $500 \mu \mathrm{l}$ of conditioned medium prepared from human lung fibroblasts (MRC-5) was added to the lower chamber. After incubation for $24 \mathrm{~h}$ at $37^{\circ} \mathrm{C}$, non-invaded cells on the upper surface of the insert membrane were removed with a cotton swab. Invaded cells on the lower surface of the membrane were fixed with $25 \%$ methanol, stained with crystal violet, and acid-extracted with $0.1 \mathrm{~N} \mathrm{HCl}$ in methanol. The absorbance at $550 \mathrm{~nm}$ was measured.

Chemosensitivity assay. The chemosensitivity of the cells was determined using an MTT assay for assessing metabolically active cells, as previously described (18). Briefly, the cell suspension was seeded into 96-well culture plates at a density of $1 \times 10^{4}$ cells $/ 100 \mu \mathrm{l} /$ well. Then, $100 \mu \mathrm{l}$ of vehicle (cell culture medium) or various concentrations of cytotoxic agents were added, and the plates were incubated for $48 \mathrm{~h}$. Cell culture medium containing MTT (Sigma-Aldrich, St. Louis, MO, USA) was added to each well and incubated for $2 \mathrm{~h}$. The number of viable cells was assessed by determining the absorbance at $550 \mathrm{~nm}$ and subtracting the absorbance at $650 \mathrm{~nm}$ (reference wavelength). The $\mathrm{IC}_{50}$ value of each drug, defined as the concentration of the drug that results in a $50 \%$ decrease in cell viability, was extrapolated from a concentration-dependent curve of the drug. Assays were performed in quadruplicate, and data were expressed as the percentage of viability relative to the control. 
Table I. Primers used for quantitative real-time RT-PCR analysis.

\begin{tabular}{lll}
\hline Gene name & \multicolumn{1}{c}{ Forward sequence 5'-3' } & \multicolumn{1}{c}{ Reverse sequence 5'-3' } \\
\hline uPA & TCGTCTGTTCCCTCCAAGGC & TGCGGATCCAGGGTAAGAAG \\
uPAR & TAAGACCAACGGGGATTGCC & TCTCCTTCTTCCCACAAGCG \\
MMP2 & CAAGGACCGGTTCATTTGGC & GGCCTCGTATACCGCATCAA \\
MMP7 & AGTGGTCACCTACAGGATCG & GGGATCTCTTTGCCCCACAT \\
MMP9 & AAGGATGGGAAGTACTGGCG \\
MMP11 & ACCTTTACTGAGGTGCACGAG & GCTCCTCAAAGACCGAGTCC \\
MMP13 & CTTAGAGGTGACTGGCAAAC & CAAATTCATGGGCTGCCACC \\
HGF & CGACAGTGTTTCCCT TCTCG & GCCCATCAAATGGGTAGAAG \\
CXCR4 & ACTTCAGTTTGTTGGCTGCGGC & ATTGAGAACCTGTTTGCGTTTCT \\
MRP-3 3 & GTCTTTGGTGCCATGGCCGT & ACCGCTGGTTCTCCAGATGCG \\
RPS13 & GGGACCCTGCGCATGAACCTG & ATGTCCGGTCGGGTGGGATA \\
\end{tabular}

Cell cycle assay. Cell cycle distribution was analyzed using a Muse $^{\mathrm{TM}}$ Cell Analyzer (Merck Millipore, Hayward, CA, USA). The Muse ${ }^{\mathrm{TM}}$ Cell Cycle Assay kit (Merck Millipore) was used according to the manufacturer's protocol. Briefly, cell pellets from each condition were washed three times with PBS and fixed in ice-cold $70 \%$ ethanol overnight at $-20^{\circ} \mathrm{C}$ prior to staining. Cells were washed again with PBS, stained with $200 \mu \mathrm{l}$ of Muse ${ }^{\mathrm{TM}}$ Cell Cycle reagent, incubated in the dark for $30 \mathrm{~min}$ at room temperature and then processed for cell cycle analysis. Results were expressed as a percentage of the cells in the G0/G1, S and G2/M phases based on differential DNA content. Experiments were performed in triplicate.

Reverse transcription-quantitative polymerase chain reaction (RT-qPCR). Gene expression levels were determined by RT-qPCR. Cells were harvested and total RNA isolation was performed using the RNeasy Mini kit (Qiagen, Inc., Valencia, CA, USA), following the manufacturer's instructions. Approximately $2 \mu \mathrm{g}$ of total RNA was reverse transcribed to cDNA with SuperScript ${ }^{\circledR}$ III Reverse Transcriptase (Invitrogen; Thermo Fisher Scientific, Inc.). RT-qPCR was performed using KAPA SYBR ${ }^{\circledR}$ FAST qPCR kits (Kapa Biosystems, Inc., Woburn, MA, USA) and a StepOnePlus $^{\text {TM }}$ Real-Time PCR system (Applied Biosystems; Thermo Fisher Scientific, Inc., Foster City, CA, USA) with the following steps: $95^{\circ} \mathrm{C}$ for $10 \mathrm{~min}$, followed by 40 cycles at $95^{\circ} \mathrm{C}$ for $15 \mathrm{sec}, 60^{\circ} \mathrm{C}$ for $30 \mathrm{sec}$ and $72^{\circ} \mathrm{C}$ for $30 \mathrm{sec}$. All primers used to amplify genes are listed in Table I. The level of gene expression relative to an internal control, RPS13 (ribosomal protein S13), was determined using the $2^{-\Delta \Delta \mathrm{Cq}}$ method (19).

Western blot analysis. The expression levels of the signaling proteins were determined by western blot analysis. Cells cultured on plastic plates or embedded in semi-solid Matrigel were harvested, and then lysed in cell signaling lysis buffer (Merck Millipore) containing protease inhibitors and phosphatase inhibitors $\left(1 \mathrm{mM} \mathrm{Na} \mathrm{VO}_{4}, 10 \mathrm{mM} \mathrm{NaF}\right.$ and $20 \mathrm{mM} \beta$-glycerophosphate). Cell extracts were resolved by $10 \%$ SDS-PAGE and transferred to Immobilon-P Transfer Membranes (EMD Millipore, Bedford, MA, USA). After blocking with $3 \%$ bovine serum albumin in Tris-buffered saline containing $0.1 \%$ Tween-20 at room temperature for $1 \mathrm{~h}$, the membranes were probed with the following primary antibodies overnight at $4^{\circ} \mathrm{C}$ : Akt $(1: 1,000$; rabbit, polyclonal; cat. no 9272), phospho-Akt (1:500; rabbit, polyclonal; cat. no. 9271), ERK (1:4,000; rabbit, polyclonal; cat. no. 9102), phospho-ERK (1:10,000; rabbit, polyclonal; cat. no. 9101), FAK (1:2,000; rabbit, polyclonal; cat. no. 3285), phospho-FAK (1:1,000; rabbit, monoclonal; cat. no. 8556), STAT3 (1:3,000; rabbit, polyclonal; cat. no. 9132), phospho-STAT3 (1:500; rabbit, monolonal; cat. no. 9145), p38 (1:1,000; rabbit, polyclonal; cat. no. 9212), phospho-p38 (1:500; rabbit, polyclonal; cat. no. 9211), p65 (1:1,000; rabbit, polyclonal; cat. no. 3034), phospho-p65 (1:500; rabbit, polyclonal; cat. no. 3031), cyclin D1 (1:1,000; rabbit, polyclonal; cat. no. 2922), p21 (1:2,000; rabbit, monoclonal; cat. no. 2947), Bcl-xL (1:10,000; rabbit, monoclonal; cat. no. 2764), Bax (1:5,000; rabbit, polyclonal; cat. no. 2772), IкB (1:1,000; rabbit, monoclonal; cat. no. 4812) (all from Cell Signaling Technology, Danvers, CA, USA), uPAR (1:500; rabbit, polyclonal; cat. no. sc-10815; Santa Cruz Biotechnology, Inc., Santa Cruz, CA, USA), MYLK (1:500; mouse, monoclonal; cat. no. sc-365352; Santa Cruz Biotechnology, Inc.) and GAPDH (1:50,000; rabbit, monoclonal; cat. no. ab190480; Abcam, Cambridge, MA, USA). Following incubation with the appropriate horseradish peroxidase-conjugated secondary antibody (1:5,000; goat, monoclonal; cat. no. 7074; Cell Signaling Technology, Danvers, CA, USA; or 1:5,000; rabbit, polyclonal; cat. no. P0260; Dako; Agilent Technologies, Inc., Santa Clara, CA, USA), the protein bands were visualized using enhanced chemiluminescence reagents.

Statistical analysis. All experiments were performed in triplicate, and the data are presented as the mean \pm standard deviation. Statistical analysis was performed using the Student's $\mathrm{t}$-test. $\mathrm{P}<0.05$ was considered to indicate a statistically significant difference.

\section{Results}

Morphology of A549 cells grown on plastic plates vs. in semi-solid Matrigel. When A549 cells plated on plastic 


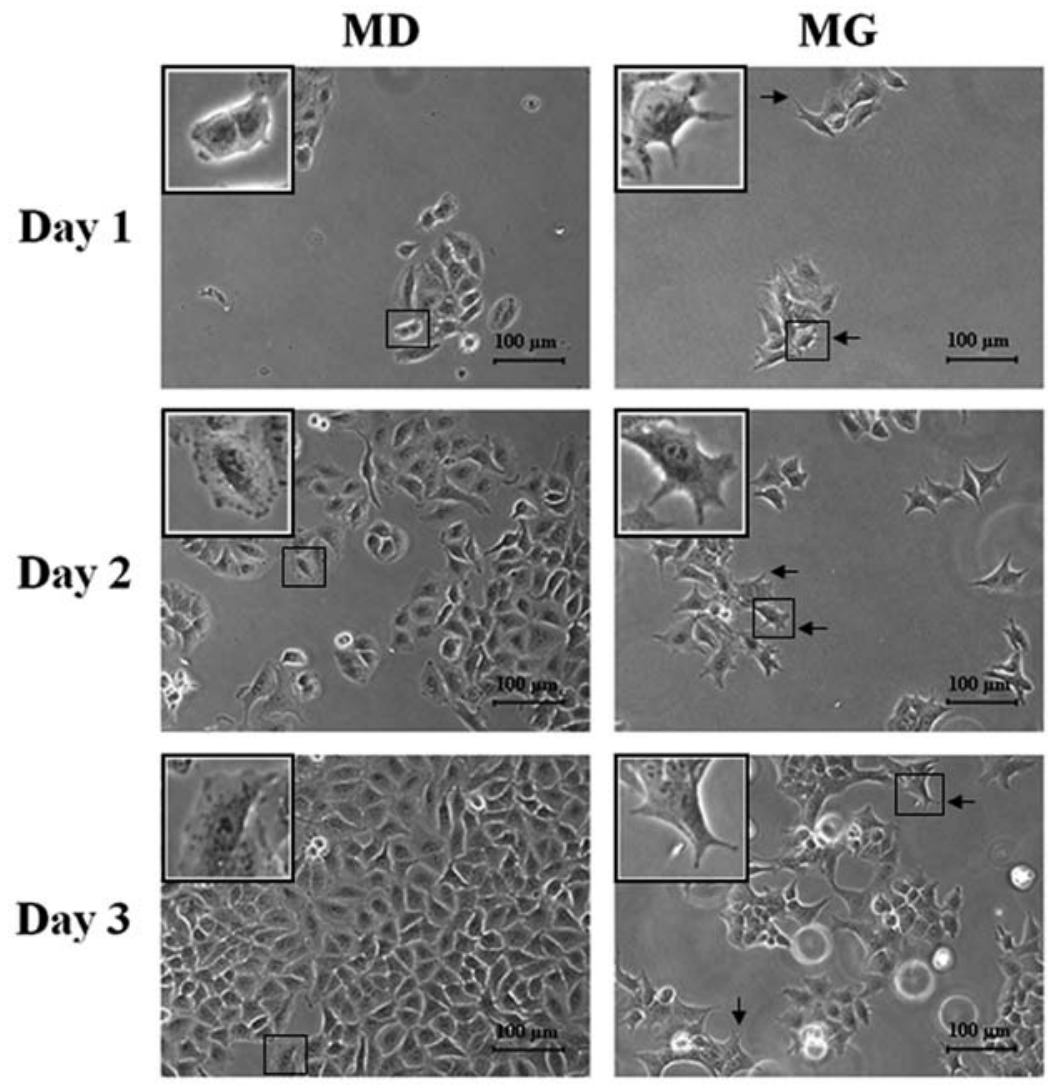

Figure 1. Morphological comparison of A549 cells cultured on plastic plates and embedded in semi-solid Matrigel. A549 cells were seeded at $5 \times 10^{4}$ cells $/ \mathrm{ml}$ in 24 well plates, either on plastic (MD) or embedded in semi-solid Matrigel (MG), and examined every $24 \mathrm{~h}$ for 3 days by inverted phase-contrast microscopy with a digital camera. A549 cells cultured on plastic plates maintained the typical cobblestone shape, while the cells cultured in semi-solid Matrigel adopted a branched morphology. Arrows indicate the branching structures protruding from the membrane of A549 cells embedded in semi-solid Matrigel. Images were captured at a magnification of $\mathrm{x} 200$. Scale bar, $100 \mu \mathrm{m}$.

dishes or embedded in semi-solid Matrigel were examined by inverted phase-contrast microscopy, the cells grown within the Matrigel matrix had undergone morphological changes by $24 \mathrm{~h}$ after plating (Fig. 1), transforming from their typical shape to branched cells. This altered morphology could be reversed by replating the semi-solid Matrigel embedded cells back into plastic plates (data not shown).

Proliferation of A549 cells grown on plastic plates vs. in semi-solid Matrigel. Proliferation of A549 cells cultured on plastic plates or embedded in semi-solid Matrigel was examined on days $0,2,4,7$ and 9 via DNA quantification using a PicoGreen DNA assay. The data revealed that the growth dynamics differed between the two cell populations; proliferative and quiescent growth characteristics were observed. Proliferative growth characteristics were observed in cells cultured on plastic plates, while quiescent growth was observed in cells cultured in semisolid Matrigel (Fig. 2). A549 cells grown within the Matrigel matrix exhibited a small increase in the number of cells during the 9 days of culture, while the number of the cells grown on plastic plates had increased 4 -fold by day 4 .

Semi-solid Matrigel embedding induces G0/G1 cell cycle arrest in A549 cells. To assess possible mechanisms underlying Matrigel-mediated cell growth inhibition, the cell cycle distribution of A549 cells cultured on plastic plates and in semi-solid Matrigel was investigated. The results demonstrated

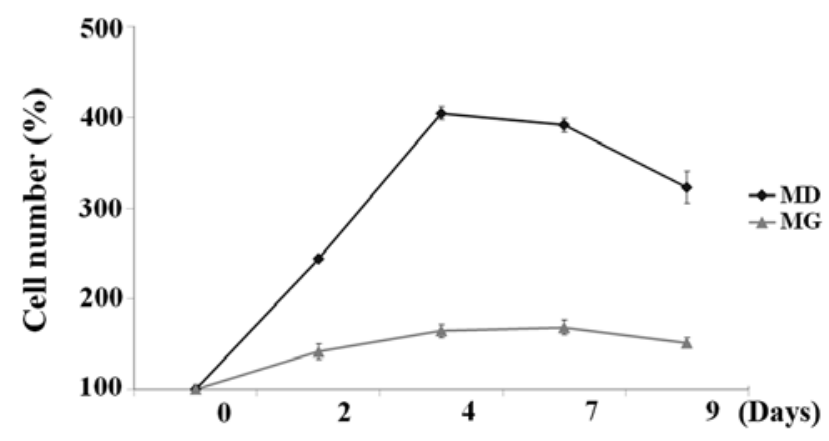

Figure 2. Effects of ECM proteins on cell proliferation. A549 cells were cultured on plastic plates or embedded in semi-solid Matrigel. Cell numbers were quantitatively measured on days $0,2,4,7$ and 9 using a PicoGreen DNA assay. The growth of A549 cells cultured in semi-solid Matrigel (MG) was greatly attenuated compared with cells cultured on plastic plates (MD).

that cells embedded in semi-solid Matrigel exhibited a higher G0/G1 population compared with cells cultured on plastic plates $(80.1 \pm 2.2$ vs. $60.1 \pm 1.5 \%$; Fig. $3 \mathrm{~A}$ and B). Consistent with this result, Matrigel embedding caused a decrease in the proportion of cells in the S phase $(6.9 \pm 0.8 \%)$ and $\mathrm{G} 2 / \mathrm{M}$ phase $(12.7 \pm 1.7 \%)$ of the cell cycle compared with cells grown on plastic plates (17.5 \pm 0.4 and $22.1 \pm 1.0 \%$, respectively). These data revealed that Matrigel embedding induced cell cycle arrest (cellular dormancy) at the G0/G1 phase, resulting in growth inhibition of A549 cells. 
Table II. Chemosensitivity of A549 cells cultured on plastic plates (MD) or in semi-solid Matrigel (MG) against various anticancer agents.

\begin{tabular}{lccc}
\hline & \multicolumn{2}{c}{$\mathrm{IC}_{50}$ values } & Fold change \\
\cline { 2 - 3 } Anticancer agents & $\mathrm{MD}$ & $\mathrm{MG}$ & $10.6 \pm 0.8$ \\
\hline Etoposide $(\mu \mathrm{M})$ & $35.3 \pm 3.3$ & $371.7 \pm 15.5$ & $5.7 \pm 1.6$ \\
Paclitaxel $(\mathrm{nM})$ & $79.8 \pm 17.7$ & $430.0 \pm 21.6$ & $4.4 \pm 0.4$ \\
Vinblastine $(\mathrm{nM})$ & $18.3 \pm 1.9$ & $79.8 \pm 5.3$ & $4.1 \pm 0.7$ \\
Doxorubicin $(\mu \mathrm{M})$ & $1 \pm 0.2$ & $3.8 \pm 0.2$ & $3.9 \pm 0.3$ \\
2-Deoxy-D-glucose $(\mathrm{mM})$ & $3.2 \pm 0.6$ & $12.2 \pm 1.6$ & $1.5 \pm 0.1$ \\
Curcumin $(\mu \mathrm{M})$ & $29.9 \pm 5.4$ & $44.5 \pm 5.9$ & $0.9 \pm 0.2$ \\
Bay 11-7085 $(\mu \mathrm{M})$ & $16.7 \pm 1.0$ & $15.2 \pm 2.7$ & \\
\hline
\end{tabular}
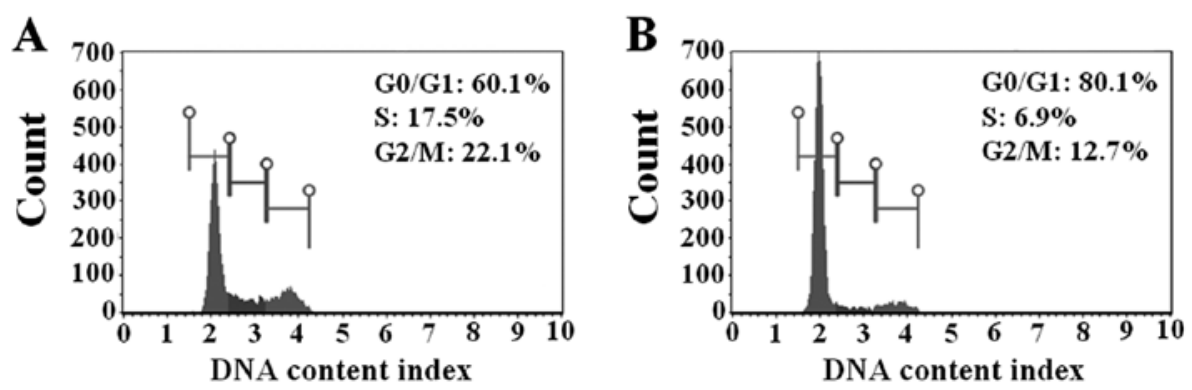

Figure 3. Analysis of cell cycle distribution of A549 cells. The percentage of cells at the G0/G1, S and G2/M phases was determined using a Muse ${ }^{\mathrm{TM}}$ Cell Analyzer. (A) Cell cycle profiles of A549 cells cultured on plastic plates. (B) Cell cycle profiles of A549 cells cultured in semi-solid Matrigel. Data from one representative experiment is shown. The experiment was performed in triplicate.

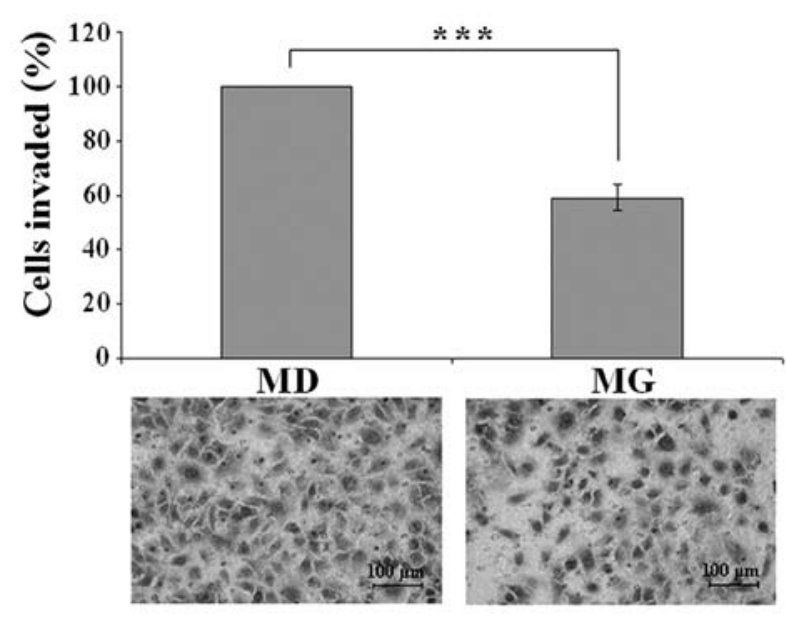

Figure 4. Effects of ECM proteins on the invasion of A549 cells. The cells were cultured on plastic plates or in semi-solid Matrigel before being subjected to Transwell invasion assays. Cells were allowed to invade towards a chemoattractant for $24 \mathrm{~h}$. Representative images of crystal violet staining of invasive A549 cells on the membrane are shown. Bar graphs illustrate quantification of the invaded cells by solubilization of crystal violet and colorimetric measurement of OD at $550 \mathrm{~nm}$. The percentage of invasive A549 cells was significantly decreased in cells cultured in semi-solid Matrigel (MG), compared with cells cultured on plastic plates (MD). ${ }^{* * *} \mathrm{P}<0.001$ Images were captured at a magnification of $\mathrm{x} 200$. Scale bar, $100 \mu \mathrm{m}$.

Effect of semi-solid Matrigel embedding on the chemosensitivity of A549 cells. Drug sensitivities of A549 cells cultured on plastic plates and embedded in semi-solid Matrigel were explored. Multiple anticancer drugs commonly used to treat a variety of cancers were used in this study. The harvested cells from both culture conditions were treated with various concentrations of the drugs for $48 \mathrm{~h}$, then MTT assays were performed to determine the survival rates. The drug concentration causing a $50 \%$ decrease in the number of cells $\left(\mathrm{IC}_{50}\right)$ was extrapolated. As presented in Table II, the results demonstrated that cell culture in semi-solid Matrigel caused a marked increase in $\mathrm{IC}_{50}$ for anticancer drugs that target actively dividing cells [etoposide (10.6-fold), paclitaxel (5.7-fold), vinblastine (4.4-fold), doxorubicin (4.1-fold) and 2-deoxy-D-glucose (3.9-fold)], as compared with cells cultured on plastic plates. This indicated a higher degree of chemoresistance in cells cultured in semi-solid Matrigel. However, the responses of the two cell populations to cytotoxic agents that are not related to cell proliferation rate were similar, such as curcumin (1.5-fold increase in $\mathrm{IC}_{50}$ in cells cultured in Matrigel) and Bay 11-7085 (0.9-fold increase; Table II). These results indicated that the chemoresistance of semi-solid Matrigel-embedded cells may be the result of a decreased rate of cell proliferation.

Effect of semi-solid Matrigel embedding on invasion. A Transwell invasion assay was performed to assess the effect of semi-solid Matrigel embedding on cell invasiveness. A549 cells were cultured on plastic plates or in semi-solid Matrigel for $72 \mathrm{~h}$, then the cells were harvested and subjected to an invasion assay. Photography and quantitative analysis of the results revealed a significant decrease in the invasion rate of cells cultured in semi-solid Matrigel (Fig. 4). The number of 
A

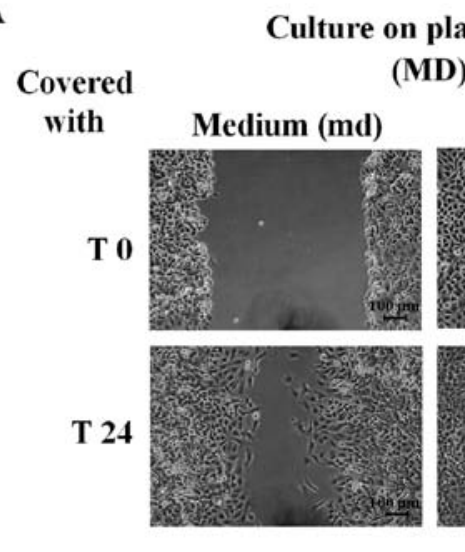

B

\author{
Embedded in Matrigel
}

(MG)
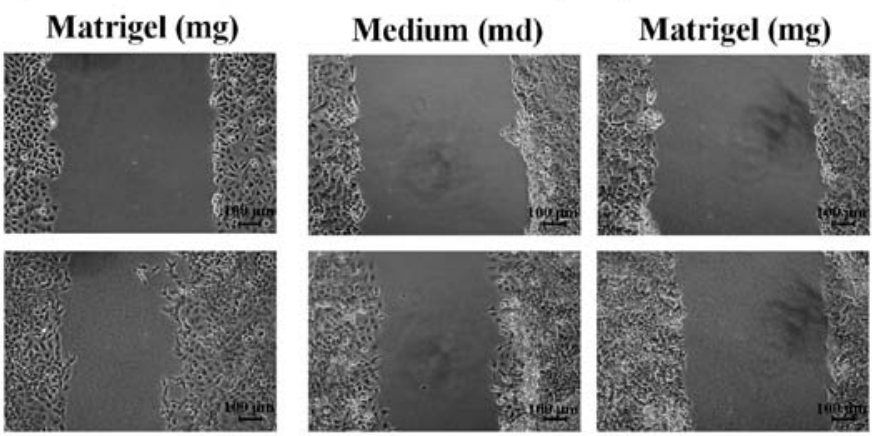

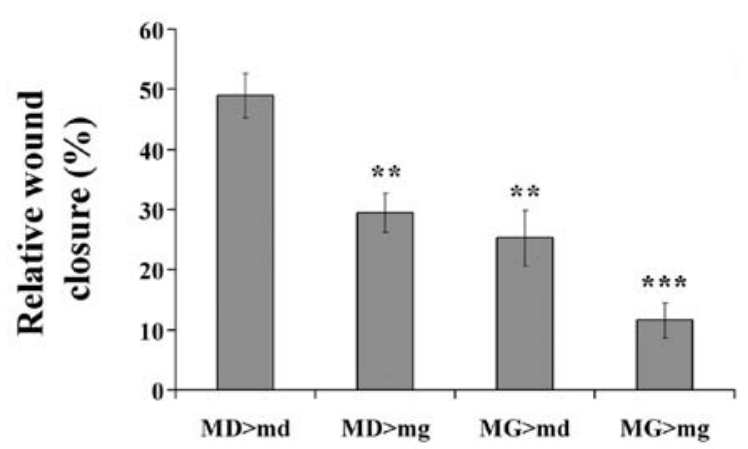

Figure 5. Effects of ECM proteins on the motility of A549 cells. Confluent monolayers of A549 cells cultured on plastic plates (MD) or in semi-solid Matrigel (MG) were scratched using a pipette tip and then covered with culture medium (md) or semi-solid Matrigel (mg). Images were obtained at 0 and $24 \mathrm{~h}$ after scratching. Quantification of the wound closure during $24 \mathrm{~h}$ of cell migration revealed that Matrigel suppresses the motility of A549 cells, resulting in reduced gap closure. ${ }^{* *} \mathrm{P}<0.01,{ }^{* * * *} \mathrm{P}<0.001$. Images were captured at a magnification of $\mathrm{x} 100$. Scale bar, $100 \mu \mathrm{m}$.

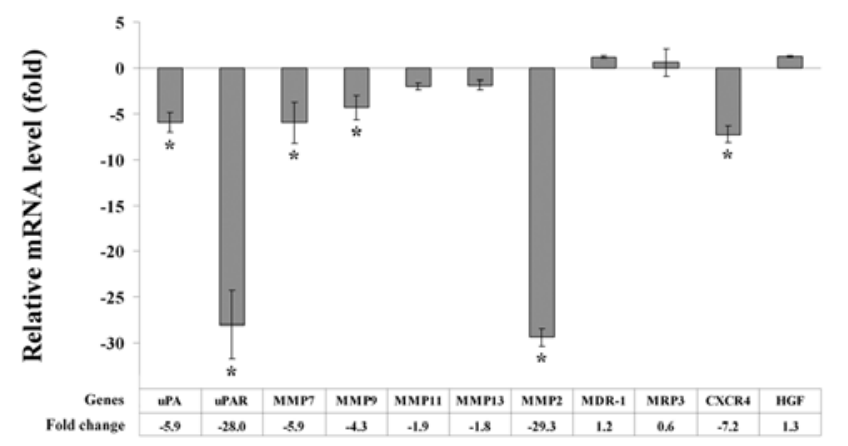

Figure 6. RT-qPCR analysis of the gene expression in A549 cells cultured in semi-solid Matrigel or on plastic plates. Relative quantification was calculated from the $\mathrm{Ct}$ values. Data are expressed as fold upregulation relative to cells cultured on plastic plates. The expression of uPA, uPAR, and several genes associated with invasion (MMP2, MMP7, MMP9 and CXCR4) was significantly decreased in A549 cells cultured in semi-solid Matrigel. The relative level of gene expression was calculated using the comparative $\mathrm{Ct}$ method, normalized to the expression of the reference gene RPS13. "Significant difference ( $\geq 3$-fold).

invading cells was $41 \%$ lower among cells cultured in semisolid Matrigel, as compared with those grown on plastic plates $(\mathrm{P}<0.001)$.

Effect of semi-solid Matrigel embedding on cell migration. A wound-healing assay was performed to investigate the effect of semi-solid Matrigel embedding on cell motility. Cell migration was expressed as a percentage of wound closure. The results demonstrated that, at $24 \mathrm{~h}, \sim 50 \%$ of the gap had closed for A549 cells grown on plastic plates and covered with culture medium, while only $30 \%$ of the initial gap had closed for cells grown on plastic plates and covered with semi-solid Matrigel (Fig. 5A). For A549 cells grown within semi-solid Matrigel, only 25 and $10 \%$ of the gap had closed when covered with culture medium and semi-solid Matrigel, respectively (Fig. 5B). These results revealed that A549 cells cultured on plastic plates exhibited higher motility compared with cells grown within semi-solid Matrigel in both conditions. In addition, cell motility was decreased in the presence of Matrigel.

Effect of semi-solid Matrigel embedding on gene expression. It has previously been reported that gene expression is altered upon cell contact with ECM proteins (4). Therefore, we investigated the consequences of semi-solid Matrigel embedding on the expression patterns of genes associated with invasion (MMP2, MMP7, MMP9, MMP11, MMP13, HGF and CXCR4) and drug resistance (MDR-1 and MRP3). In addition, genes that are associated with tumor progression and metastasis [uPA and uPA receptor (uPAR)] were analyzed. RT-qPCR was performed to determine the level of gene expression. Gene expression was normalized against RPS13 expression. Using a threshold value of 3 -fold expression change, the results revealed that A549 cells cultured in semi-solid Matrigel exhibited decreased expression levels of uPA, uPAR and multiple genes associated with invasion (MMP2, MMP7, MMP9 and CXCR4), as compared with cells cultured on plastic plates. However, no notable differences were observed in the expression levels of drug resistance genes, MDR-1 and MRP3 (Fig. 6). 


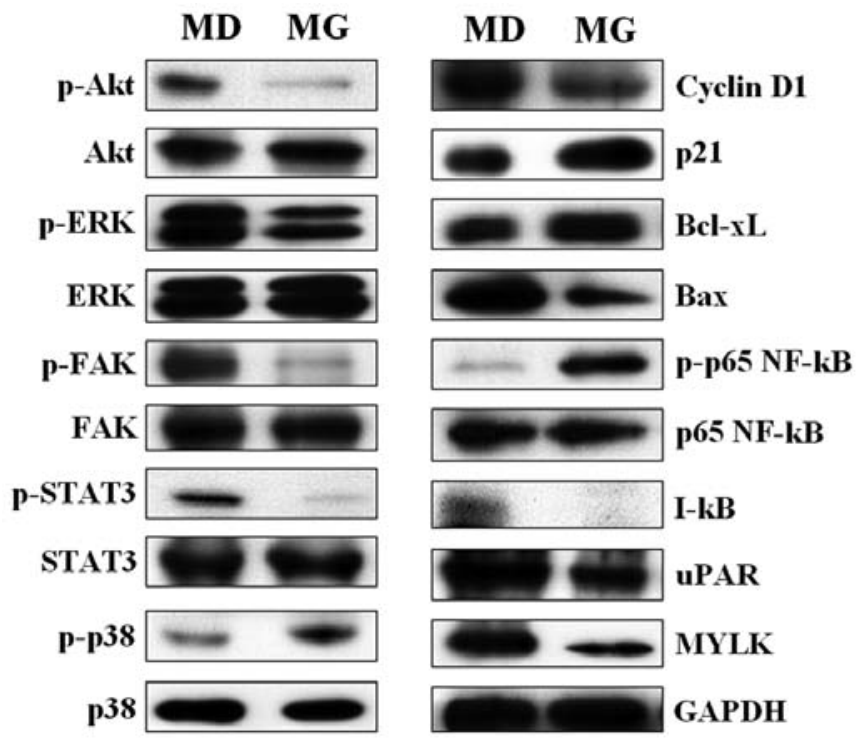

Figure 7. Western blot analysis of total extracts from A549 cells cultured in semi-solid Matrigel (MG) or on plastic plates (MD). Whole cell extracts $(40 \mu \mathrm{g})$ were prepared and subjected to immunoblotting using the indicated monoclonal antibodies. Representative blots from at least three separate experiments are shown. GAPDH was used as the loading control.

Effect of semi-solid Matrigel embedding on protein expression. Protein expression levels of signaling molecules associated with cell proliferation, metastasis, chemoresistance and apoptosis were assessed. Western blot analysis of proteins extracted from A549 cells indicated that cells grown within semi-solid Matrigel had reduced expression of p-ERK, p-Akt and p-STAT3 (positive regulators of cell growth, proliferation and survival), compared with cells cultured on plastic plates (Fig. 7). Bcl-xL, an anti-apoptotic protein, was up-regulated in cells cultured in semi-solid Matrigel, while a decreased level of the pro-apoptotic protein, Bax, was detected (Fig. 7). An increased level of p-p65 (NF- $\mathrm{kB}$ ) protein and decreased levels of $\mathrm{I} \kappa \mathrm{B}$ were observed in cells cultured in semi-solid Matrigel (Fig. 7), suggested that NF- $\mathrm{BB}$ signaling was activated in the Matrigel-embedded cells. Furthermore, the upregulation of p-p38 and p21 was observed in cells cultured in semi-solid Matrigel, while the expression of p-FAK, cyclin D1, uPAR and myosin light-chain kinase (MYLK) was decreased, compared with cells cultured on plastic plates (Fig. 7). These findings revealed that changes in the expression of signaling molecules in cells cultured in semi-solid Matrigel shifted the cells from a highly proliferative to a less proliferative state, and also enhanced apoptotic resistance.

Inhibition of MAPK and Akt pathways induces G0/G1 cell cycle arrest and protects against etoposide-induced cell death. Specific kinase inhibitors of MEK1/2 (U0126), PI3K (LY-294002) and JAK2 (AG-490) were used to investigate whether these signaling pathways are associated with Matrigelmediated dormancy and chemoresistance. First, the effects of U0126, LY-294002 and AG-490 on cell viability were evaluated. A549 cells were treated with various concentrations of U0126, LY-294002 and AG-490, after which an MTT assay was performed to determine cytotoxicity following treatment. Inhibitor concentrations that resulted in $>80 \%$ cell viability were used. Pretreatment for $24 \mathrm{~h}$ with $30 \mu \mathrm{M}$ of U0126, $20 \mu \mathrm{M}$ of LY-294002 and $30 \mu \mathrm{M}$ of AG-490 did not significantly affect A549 cell viability (data not shown). Western blot analysis revealed decreased phosphorylation of ERK1/2, Akt and STAT3 following treatment with $30 \mu \mathrm{M}$ of U0126, $20 \mu \mathrm{M}$ of LY-294002 and $30 \mu \mathrm{M}$ of AG-490, respectively, for $24 \mathrm{~h}$ (data not shown). Cell cycle analysis revealed that blockade of ERK1/2 and Akt with U0126 and LY-294002 resulted in G0/G1 phase cell cycle arrest, similar to that observed in cells embedded in semi-solid Matrigel (Table III). Treatment with AG-490 had no notable effect on the cell cycle distribution of A549 cells (Table III).

To determine whether the decrease in phosphorylation of ERK1/2, Akt and STAT3 mediates chemoresistance of semi-solid Matrigel-embedded cells, chemosensitivity to etoposide and Bay 11-7085 was evaluated in the presence of the specific kinase inhibitors. Etoposide and Bay 11-7085 were used as representative examples of drugs that are associated and not associated with, respectively, the cell proliferation rate. The cells were pre-incubated with the kinase inhibitors for $24 \mathrm{~h}$ prior to and during the chemosensitivity assay. Comparing the effects on chemosensitivity, U0126 and LY-294002 were determined to increase etoposide resistance, similar to that observed in cells cultured in semi-solid Matrigel (the $\mathrm{IC}_{50}$ value of cells treated with U0126 and LY-294002 was $299 \pm 23.3 \mu \mathrm{M}$ and $243.3 \pm 16.5 \mu \mathrm{M}$, respectively; Fig. 8). However, the chemoresistance to etoposide was unaffected by STAT3 inhibition with AG-490 (the $\mathrm{IC}_{50}$ value of cells pre-treated with AG-490 was 30.8 $\pm 1.2 \mu \mathrm{M}$ ). For Bay 11-7085, treatment with U0126, LY-294002 and AG-490 exhibited no effect on chemosensitivity (the $\mathrm{IC}_{50}$ value of cells treated with U0126, LY-294002 and AG-490 were 19.0 $\pm 0.5,19.4 \pm 0.1$ and

Table III. Cell cycle progression of A549 cells.

Cell cycle distribution (\%)

Treatment

\begin{tabular}{crc}
\hline G0/G1 & \multicolumn{1}{c}{$\mathrm{S}$} & $\mathrm{G} 2 / \mathrm{M}$ \\
\hline $60.1 \pm 1.5$ & $17.5 \pm 0.4$ & $22.1 \pm 1.0$ \\
$80.1 \pm 2.2$ & $6.9 \pm 0.8$ & $12.7 \pm 1.7$ \\
$77.0 \pm 1.5$ & $10.8 \pm 0.7$ & $11.9 \pm 1.1$ \\
$78.4 \pm 1.3$ & $9.5 \pm 0.6$ & $12.0 \pm 0.8$ \\
$61.3 \pm 2.8$ & $16.5 \pm 1.4$ & $21.8 \pm 1.4$
\end{tabular}

Cultured on plastic plates

Embedded in semi-solid Matrigel

U0126 (30 $\mu \mathrm{M})$

LY-294002 $(20 \mu \mathrm{M})$

AG-490 $(30 \mu \mathrm{M})$ 


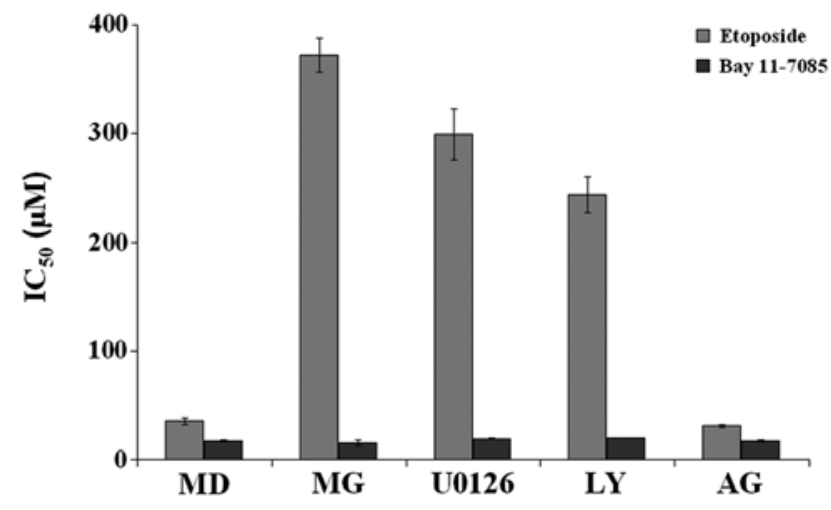

Figure 8. Effects of specific kinase inhibitors (U0126, LY-294002 and AG-490) on chemosensitivity to etoposide and Bay 11-7085. Cells were preincubated with U0126 $(30 \mu \mathrm{M})$, LY-294002 $(20 \mu \mathrm{M})$ and AG-490 $(30 \mu \mathrm{M})$ $24 \mathrm{~h}$ prior to and during the chemosensitivity assay. Cells were then treated with various concentrations of the drugs for $48 \mathrm{~h}$ and cytotoxicity was evaluated using an MTT assay.

17.0 $\pm 1.1 \mu \mathrm{M}$, respectively; Fig. 8). These findings were in agreement with those of the cell cycle distribution experiment, suggesting that inhibition of ERK1/2 and Akt with U0126 and LY-294002 results in cell dormancy (Table III), which leads to enhanced chemoresistance for the drugs that target actively proliferating cells. These data revealed that Matrigel-mediated growth inhibition of A549 cells and protection from chemotherapy-induced apoptosis occurred predominantly via the MAPK and Akt pathways.

\section{Discussion}

Metastasis is the prime cause of cancer-related deaths. Many cancer patients who have no clinical symptoms after primary tumor removal suffer from disease relapse years or decades later $(20,21)$. A likely explanation for cancer recurrence after a long latent period is that metastatic cells remain dormant in the body, resistant to current therapies and begin reactivation in a permissive microenvironment (22). Various factors have been suggested as possible contributors to cell dormancy, including complex interactions between metastatic cells and the microenvironment (23). Interactions between metastatic cancer cells and the new organ microenvironment have been demonstrated to influence the proliferative properties of cancer cells at the metastatic site $(24,25)$. Recent experiments have indicated that cell-ECM interactions inhibit the proliferation of metastatic melanoma cells $(26,27)$.

Matrigel is a commercially available matrix resembling the complex tissue microenvironment. Matrigel contains various soluble factors, including insulin-like growth factor, TGF- $\beta$, tissue plasminogen activator, EGF, bFGF, and other growth factors found in EHS tumors. Increasingly, cell-ECM interactions are being modeled to bridge the gap between in vitro and in vivo experiments. For instance, it has been reported that MCF-7 and MDA-MB-231 cells cultured in ECM exhibited growth characteristics that correspond with their behavior at secondary sites in vivo (13). Vascular smooth muscle cells grown on Matrigel were morphologically similar to those found in in vivo studies of contractile vascular smooth muscle (28). This was consistent with another study detailing that three 21T cell lines grown in Matrigel exhibited morphological and functional characteristics resembling in vivo behavior, including colony morphology, cell polarization, acinar structure formation, cell proliferation and invasion (29). The present results revealed that A549 cells grown within semi-solid Matrigel exhibited some characteristics similar to those found in dormant cancer cells, including a decrease in cell proliferation, cell motility and invasion, and an increase in chemoresistance. Cell cycle distribution analysis revealed that growth of A549 cells within semi-solid Matrigel matrix induced G0/G1 cell cycle arrest.

MAPK/ERK, PI3K/Akt and STAT3 have been implicated in cellular growth, proliferation and survival. The results of the present study revealed a marked reduction in p-ERK, p-Akt and p-STAT3 expression, concurrently with a decreased proliferation rate of A549 cells cultured in semisolid Matrigel, compared with A549 cells cultured on plastic plates. The reduction in ERK1/2 activation has been linked to tumor dormancy in vivo $(30,31)$. The activation of $\mathrm{p} 38$ stress-activated kinase induced cell cycle arrest via inhibition of ERK1/2 signaling and UPAR expression (30), consistent with the enhanced phosphorylation of p38 MAPK and the reduction of UPAR at both mRNA and protein levels observed in our results. UPA and its receptor, UPAR, have been associated with metastasis, tumor progression and reduced overall survival of patients (32). Current evidence demonstrates that downregulation of UPAR leads to a reduction in FAK phosphorylation and deactivation of ERK $1 / 2$, resulting in dormancy in vivo $(22,30,33)$. The decrease in FAK phosphorylation and uPA mRNA levels in our study further support this notion. In support of a role for FAK and MYLK in cell migration, the low-motility A549 cells cultured in semi-solid Matrigel exhibited a decrease in both MYLK and p-FAK protein levels. A recent study revealed that MYLK also has a functional role in cell proliferation, and inhibition of MYLK prevents the transition from dormant to proliferative state (13). The expression level of genes linked to cancer cell invasion (MMP2, MMP7, MMP9 and CXCR4) were also decreased in cells cultured in semi-solid Matrigel compared with cells cultured on plastic plates, which was consistent with the decrease in the invasion rate of cells cultured in semi-solid Matrigel.

Cyclin D1 facilitates cell cycle progression by sequestering the CDK inhibitor $\mathrm{p} 21$, allowing $\mathrm{S}$ phase initiation and progression. Consistent with the decrease in proliferative activity of A549 cells embedded in semi-solid Matrigel, the protein level of cyclin D1 was decreased concurrently with an increased p21 level, thus preventing cells from reentering the cell division cycle. According to a recent study, a decrease in p-STAT3 and p-ERK expression led to downregulation of the downstream target molecules, including cyclin D1 (34). p21 has been previously demonstrated to be negatively regulated by Akt (35), so the inactivation of Akt further promotes the inhibitory effect of p21 on cell cycle progression. Changes in the levels of these signaling molecules are likely to be responsible for dormancy of A549 cells cultured in semi-solid Matrigel.

A number of studies have presented evidence for ECM-mediated cytoprotection (36-38). The response of metastases in different organ microenvironments to chemotherapy differs markedly due to interaction of metastatic cells with local environmental factors (39). Soluble cytokines secreted by 
the tumor microenvironment may activate signal transduction pathways that influence the response to cytotoxic drugs of tumor cells (40). The concept of ECM-mediated chemoresistance is also supported by the observation that adhesion of small cell lung cancer (SCLC) cells to laminin enhances resistance to several cytotoxic drugs (1). Etoposide treatment of SCLC cells grown on fibronectin significantly reduced caspase-3 activation and apoptosis (41). The ECM-mediated chemoresistance in the present study was likely to be due to a decrease in cell proliferation, since A549 cells embedded in semi-solid Matrigel exhibited marked resistance to chemotherapy drugs that were related to the cell proliferation rate, compared with cells cultured on plastic plates. By contrast, the cells from both culture conditions exhibited similar chemosensitivity for drugs that were not related to the cell proliferation rate. Thus, targeting ECM-suppressed cells with compounds that are more potent against cells cultured in semi-solid Matrigel could represent a possible direction for cancer treatment.

Upregulation of $\mathrm{Bcl}-\mathrm{xL}$ and downregulation of $\mathrm{Bax}$ may also be involved, at least in part, in the evasion of apoptosis of A549 cells cultured in semi-solid Matrigel. In addition, p-p65 has been reported to confer resistance to chemotherapy (42). An increased level of p-p65, concurrent with a decreased level of $\mathrm{I} \kappa \mathrm{B}$, could also be responsible for the acquired chemoresistance of A549 cells cultured in semisolid Matrigel. Overexpression of drug efflux pumps has been associated with chemoresistance (43). However, the mRNA expression levels of MDR-1 (encoding P-glycoprotein) and MRP3 (encoding multi-drug resistance protein) was unaffected by ECM proteins in the present study. Recent studies have established that cancer stem cells (CSCs), a distinct population of cancer cells possessing stem-like properties, are responsible for tumor initiation and progression, metastasis, therapy/apoptosis resistance and relapse (44-49). CSCs and dormant cells are closely related, since stem cells remain dormant to prevent cell exhaustion and acquire mutations until they become activated in response to injury or stress (50). A549 cells embedded in semi-solid Matrigel in the present study acquired some stem-like properties, in terms of quiescence and resistance to therapy. In addition, CSCs and cells embedded in semi-solid Matrigel share some mechanisms for dormancy and chemotherapeutic resistance, including cell cycle modifications and the overexpression of anti-apoptotic proteins. Cell cycle progression analysis and cytotoxicity assays indicated that blockade of ERK1/2 and Akt in A549 cells induced G0/G1 phase cell cycle arrest, accompanied by chemoresistance, similar to that observed in cells cultured in semi-solid Matrigel. These results are in agreement with those of previous studies, which reported that the inhibition of ERK1/2 and Akt induced dormancy and was associated with acquired resistance to chemotherapy drugs $(51,52)$.

In conclusion, our results revealed that MAPK/ERK and PI3K/Akt pathways may be possible mechanisms underlying Matrigel-mediated dormancy and the chemoresistance of A549 cells. The semi-solid Matrigel culture environment used in the present study induced a dormant-like phenotype that may mimic in vivo dormancy and could be used to predict responses to chemotherapy. This semi-solid Matrigel-embedded cell culture system could provide a simple means for screening and developing new therapeutic approaches to improve the response to chemotherapeutic agents and eliminate dormant micrometastatic cells.

\section{Acknowledgements}

This study was supported by the Chulabhorn Research Institute (Bangkok, Thailand).

\section{Competing interests}

The authors declare that they have no competing interests.

\section{References}

1. Fridman R, Giaccone G, Kanemoto T, Martin GR, Gazdar AF and Mulshine JL: Reconstituted basement membrane (matrigel) and laminin can enhance the tumorigenicity and the drug resistance of small cell lung cancer cell lines. Proc Natl Acad Sci USA 87: 6698-6702, 1990.

2. Weaver VM, Fischer AH, Peterson OW and Bissell MJ: The importance of the microenvironment in breast cancer progression: Recapitulation of mammary tumorigenesis using a unique human mammary epithelial cell model and a threedimensional culture assay. Biochem Cell Biol 74: 833-851, 1996.

3. Romero-López M, Trinh AL, Sobrino A, Hatch MM, Keating MT, Fimbres C,Lewis DE, Gershon PD, Botvinick EL, Digman M, et al: Recapitulating the human tumor microenvironment: Colon tumorderived extracellular matrix promotes angiogenesis and tumor cell growth. Biomaterials 116: 118-129, 2017.

4. Cramer GM, Jones DP, El-Hamidi H and Celli JP: ECM composition and rheology regulate growth, motility, and response to photodynamic therapy in 3D models of pancreatic ductal adenocarcinoma. Mol Cancer Res 15: 15-25, 2017.

5. Lin CQ and Bissell MJ: Multi-faceted regulation of cell differentiation by extracellular matrix. FASEB J 7: 737-743, 1993.

6. Streuli $\mathrm{CH}$, Bailey $\mathrm{N}$ and Bissell MJ: Control of mammary epithelial differentiation: Basement membrane induces tissuespecific gene expression in the absence of cell-cell interaction and morphological polarity. J Cell Biol 115: 1383-1395, 1991.

7. Muschler J, Lochter A, Roskelley CD, Yurchenco P and Bissell MJ: Division of labor among the alpha6beta4 integrin, beta1 integrins, and an E3 laminin receptor to signal morphogenesis and beta-casein expression in mammary epithelial cells. Mol Biol Cell 10: 2817-2828, 1999.

8. Nakajima M, Morikawa K, Fabra A, Bucana CD and Fidler IJ: Influence of organ environment on extracellular matrix degradative activity and metastasis of human colon carcinoma cells. J Natl Cancer Inst 82: 1890-1898, 1990.

9. Gohji K, Nakajima M, Boyd D, Dinney CP, Bucana CD, Kitazana S, Kamidono S and Fidler IJ: Organ-site dependence for the production of urokinase-type plasminogen activator and metastasis by human renal cell carcinoma cells. Am J Pathol 151: 1655-1661, 1997.

10. Luzzi KJ, MacDonald IC, Schmidt EE, Kerkvliet N, Morris VL, Chambers AF and Groom AC: Multistep nature of metastatic inefficiency: Dormancy of solitary cells after successful extravasation and limited survival of early micrometastases. Am J Pathol 153: 865-873, 1998.

11. Naumov GN, MacDonald IC, Weinmeister PM, Kerkvliet N, Nadkarni KV, Wilson SM, Morris VL, Groom AC and Chambers AF: Persistence of solitary mammary carcinoma cells in a secondary site: A possible contributor to dormancy. Cancer Res 62: 2162-2168, 2002.

12. Naumov GN, Townson JL, MacDonald IC, Wilson SM, Bramwell VH, Groom AC and Chambers AF: Ineffectiveness of doxorubicin treatment on solitary dormant mammary carcinoma cells or late-developing metastases. Breast Cancer Res Treat 82: 199-206, 2003.

13. Barkan D, Kleinman H, Simmons JL, Asmussen H, Kamaraju AK, Hoenorhoff MJ, Liu ZY, Costes SV, Cho EH, Lockett S, et al: Inhibition of metastatic outgrowth from single dormant tumor cells by targeting the cytoskeleton. Cancer Res 68: 6241-6250, 2008.

14. Boyerinas B, Zafrir M, Yesilkanal AE, Price TT, Hyjek EM and Sipkins DA: Adhesion to osteopontin in the bone marrow niche regulates lymphoblastic leukemia cell dormancy. Blood 121: 4821-4831, 2013 
15. Ghajar CM, Peinado H, Mori H, Matei IR, Evason KJ, Brazier H, Almeida D, Koller A, Hajjar KA, Stainier DY, et al: The perivascular niche regulates breast tumour dormancy. Nat Cell Biol 15: 807-817, 2013.

16. Oridate N, Lotan R and Lotan D: Reconstituted basement membrane (Matrigel): A useful semisolid medium for growth of tumor cell colonies. In Vitro Cell Dev Biol Anim 32: 192-193, 1996.

17. Kleinman HK and Martin GR: Matrigel: Basement membrane matrix with biological activity. Semin Cancer Biol 15: 378-386, 2005.

18. Alley MC, Scudiero DA, Monks A, Hursey ML, Czerwinski MJ, Fine DL, Abbott BJ, Mayo JG, Shoemaker RH and Boyd MR: Feasibility of drug screening with panels of human tumor cell lines using a microculture tetrazolium assay. Cancer Res 48: 589-601, 1988

19. Livak KJ and Schmittgen TD: Analysis of relative gene expression data using real-time quantitative PCR and the $2^{-\Delta \Delta C_{\mathrm{T}}}$ method. Methods 25: 402-408, 2001.

20. Meltzer A: Dormancy and breast cancer. J Surg Oncol 43: 181-188, 1990.

21. Karrison TG, Ferguson DJ and Meier P: Dormancy of mammary carcinoma after mastectomy. J Natl Cancer Inst 91: 80-85, 1999.

22. Aguirre-Ghiso JA: Models, mechanisms and clinical evidence for cancer dormancy. Nat Rev Cancer 7: 834-846, 2007.

23. Yu W, Kim J and Ossowski L: Reduction in surface urokinase receptor forces malignant cells into a protracted state of dormancy. J Cell Biol 137: 767-777, 1997.

24. Radinsky R: Modulation of tumor cell gene expression and phenotype by the organ-specific metastatic environment. Cancer Metastasis Rev 14: 323-338, 1995.

25. Fidler IJ: Seed and soil revisited: Contribution of the organ microenvironment to cancer metastasis. Surg Oncol Clin N Am 10: 257-269, 2001.

26. Henriet P, Zhong ZD, Brooks PC, Weinberg KI and DeClerck YA Contact with fibrillar collagen inhibits melanoma cell proliferation by up-regulating p27KIP1. Proc Natl Acad Sci USA 97: 10026-10031, 2000.

27. Roth JM, Akalu A, Zelmanovich A, Policarpio D, Ng B, MacDonald S, Formenti S, Liebes L and Brooks PC: Recombinant alpha2(IV)NC1 domain inhibits tumor cell-extracellular matrix interactions, induces cellular senescence, and inhibits tumor growth in vivo. Am J Pathol 166: 901-911, 2005.

28. Li X, Tsai P, Wieder ED, Kribben A, Van Putten V, Schrier RW and Nemenoff RA: Vascular smooth muscle cells grown on Matrigel. A model of the contractile phenotype with decreased activation of mitogen-activated protein kinase. J Biol Chem 269: 19653-19658, 1994

29. Souter LH, Andrews JD, Zhang G, Cook AC, Postenka CO, Al-Katib W, Leong HS, Rodenhiser DI, Chambers AF and Tuck AB: Human 21T breast epithelial cell lines mimic breast cancer progression in vivo and in vitro and show stage-specific gene expression patterns. Lab Invest 90: 1247-1258, 2010.

30. Aguirre Ghiso JA, Kovalski K and Ossowski L: Tumor dormancy induced by downregulation of urokinase receptor in human carcinoma involves integrin and MAPK signaling. J Cell Biol 147: 89-104, 1999.

31. Aguirre Ghiso JA, Liu D, Mignatti A, Kovalski K and Ossowski L: Urokinase receptor and fibronectin regulate the ERK $^{\text {MAPK }}$ to $\mathrm{p} 38^{\text {MAPK }}$ activity ratios that determine carcinoma cell proliferation or dormancy in vivo. Mol Biol Cell 12: 863-879, 2001.

32. Pavón MA, Arroyo-Solera I, Céspedes MV, Casanova I, León X and Mangues R: uPA/uPAR and SERPINE1 in head and neck cancer: Role in tumor resistance, metastasis, prognosis and therapy. Oncotarget 7: 57351-57366, 2016.
33. Aguirre Ghiso JA: Inhibition of FAK signaling activated by urokinase receptor induces dormancy in human carcinoma cells in vivo. Oncogene 21: 2513-2524, 2002.

34. Modi PK, Komaravelli N, Singh N and Sharma P: Interplay between MEK-ERK signaling, cyclin D1, and cyclin-dependent kinase 5 regulates cell cycle reentry and apoptosis of neurons. Mol Biol Cell 23: 3722-3730, 2012.

35. Rössig L, Jadidi AS, Urbich C, Badorff C, Zeiher AM and Dimmeler S: Akt-dependent phosphorylation of $\mathrm{p} 21^{\mathrm{Cip} 1}$ regulates PCNA binding and proliferation of endothelial cells. Mol Cell Biol 21: 5644-5657, 2001.

36. Hodkinson PS, Mackinnon AC and Sethi T: Extracellular matrix regulation of drug resistance in small-cell lung cancer. Int $\mathrm{J}$ Radiat Biol 83: 733-741, 2007.

37. Said G, Guilbert M, Morjani H, Garnotel R, Jeannesson P and El Btaouri H: Extracellular matrix proteins modulate antimigratory and apoptotic effects of Doxorubicin. Chemother Res Pract 2012: 268681, 2012.

38. Majidinia M and Yousefi B: Breast tumor stroma: A driving force in the development of resistance to therapies. Chem Biol Drug Des 89: 309-318, 2017

39. Fidler IJ, Wilmanns C, Staroselsky A, Radinsky R, Dong Z and Fan D: Modulation of tumor cell response to chemotherapy by the organ environment. Cancer Metastasis Rev 13: 209-222, 1994.

40. Dalton WS: The tumor microenvironment as a determinant of drug response and resistance. Drug Resist Updat 2: 285-288, 1999.

41. Buttery RC, Rintoul RC and Sethi T: Small cell lung cancer: The importance of the extracellular matrix. Int J Biochem Cell Biol 36: 1154-1160, 2004

42. Godwin P, Baird AM, Heavey S, Barr MP, O'Byrne KJ and Gately K: Targeting nuclear factor-kappa B to overcome resistance to chemotherapy. Front Oncol 3: 120, 2013.

43. Ughachukwu P and Unekwe P: Efflux pump-mediated resistance in chemotherapy. Ann Med Health Sci Res 2: 191-198, 2012.

44. Liu H, Patel MR, Prescher JA, Patsialou A, Qian D, Lin J, Wen S, Chang YF, Bachmann MH, Shimono Y, et al: Cancer stem cells from human breast tumors are involved in spontaneous metastases in orthotopic mouse models. Proc Natl Acad Sci USA 107: 18115-18120, 2010

45. Pajonk F, Vlashi E and McBride WH: Radiation resistance of cancer stem cells: The 4 R's of radiobiology revisited. Stem Cells 28: 639-648, 2010.

46. D'Andrea FP: Intrinsic radiation resistance of mesenchymal cancer stem cells and implications for treatment response in a murine sarcoma model. Dan Med J 59: B4388, 2012.

47. LaBarge MA: The difficulty of targeting cancer stem cell niches. Clin Cancer Res 16: 3121-3129, 2010.

48. Lacerda L, Pusztai L and Woodward WA: The role of tumor initiating cells in drug resistance of breast cancer: Implications for future therapeutic approaches. Drug Resist Updat 13: 99-108, 2010.

49. Wang Z, Li Y, Ahmad A, Azmi AS, Kong D, Banerjee S and Sarkar FH: Targeting miRNAs involved in cancer stem cell and EMT regulation: An emerging concept in overcoming drug resistance. Drug Resist Updat 13: 109-118, 2010.

50. Trumpp A, Essers M and Wilson A: Awakening dormant haematopoietic stem cells. Nat Rev Immunol 10: 201-209, 2010.

51. Endo H, Okuyama H, Ohue M and Inoue M: Dormancy of cancer cells with suppression of AKT activity contributes to survival in chronic hypoxia. PLoS One 9: e98858, 2014.

52. Li Q, Chow AB and Mattingly RR: Three-dimensional overlay culture models of human breast cancer reveal a critical sensitivity to mitogen-activated protein kinase kinase inhibitors. J Pharmacol Exp Ther 332: 821-828, 2010. 